\title{
Indice de Shock: ¿Puede Predecir La Necesidad de Cirugia de Conrol de Daños En Trauma Penetrante?
}

\author{
${ }^{1}$ Carlos A Ordonez, ${ }^{2}$ Viviana Orozco, ${ }^{3}$ Juan C Puyana, ${ }^{4}$ Michael Parra, ${ }^{5}$ Paola Ossa, ${ }^{6}$ Sara S Escobar \\ ${ }^{7}$ Cecibel Cevallos, ${ }^{8}$ Alberto Garcia, ${ }^{9}$ Marisol Badiel, ${ }^{10}$ Mónica AM García
}

\section{RESUMEN}

Introducción: Valores elevados del Índice de Shock (IS) se han asociado con un incremento en la mortalidad y con la necesidad de requerimiento de transfusiones masivas en pacientes traumatizados, pero su habilidad para identificar pacientes que requieran Cirugía de Control de Daños (CCD) es desconocida. El objetivo de este estudio fue identifica un punto de corte del IS para predecir necesidad de CCD en pacientes con trauma penetrante.

Materials y métodos: Estudio retrospectivo por un periodo de 10 años que incluyo todos los pacientes adultos que ingresaron por trauma penetrante de torso a un Centro de Trauma de referencia. El IS fue calculado al ingreso y los pacientes se dividieron en los que necesitaron CCD y los que no (No-CCD). Para determinar la exactitud del IS identificando pacientes que requirieron CCD se calculo el Área Bajo la Curva ROC (AUROC) del 95\%.

Resultados: 747 pacientes fueron incluidos, $92.3 \%$ fueron hombres y el $80 \%$ sufrieron heridas por arma de fuego. El grupo de CCD tuvo un total de 331(44\%) pacientes y el de No-CCD 416 (56\%). La mediana de IS fue mayor en el grupo de CCD que en el de No-CCD (1.01 [0.76-1.5]) vs. 0.79 [0.64-1.00] ( $p=0.0001)$ ). El área bajo la curva ROC fue de 0.69 [95\% Cl 0.69-0.77].

Conclusión: EI IS puede ser en cierto grado indicativo de la necesidad de CCD en los pacientes con trauma penetrante de torso, sin embargo un punto específico de corte para predecir CCD no fue estadísticamente encontrado.

\footnotetext{
${ }^{1,3,4,8,9}$ Professor, ${ }^{2,5,6}$ Resident, ${ }^{7}$ Fellow, ${ }^{10}$ Lecturer

1,5Departamento de Cirugía, Fundación Valle del Lili, Cali Colombia

${ }^{2,6}$ Clinical Research, Fundación Valle del Lili, Cali, Valle de Cauca, Colombia

${ }^{3}$ Department of Surgery, University of Pittsburgh, Pittsburgh Pennsylvania, USA

${ }^{4}$ Department of Surgery, Broward Health Medical Center, Fort Lauderdale, Florida, USA

${ }^{7}$ Cirugia de Trauma y Emergencias, Universidad del Valle, Cali Valle del Cauca, Colombia

${ }^{8}$ Departamento de Cirugía, Universidad del Valle - Fundación Valle del Lili, Cali, Valle del Cauca, Colombia

9,10Departamento de Cirugía, Hospital Universitario del Valle Cali, Valle del Cauca, Colombia
}

Corresponding Author: Viviana Orozco, Resident, Clinical Research, Fundación Valle del Lili, Cali, Valle del Cauca Colombia, Phone: 3720074, e-mail: vorozcomartin@gmail.com
Palabras Claves: Cirugía de control de daños, Hemorragia, Sociedad panamericana de trauma, Trauma penetrante, Predicción, Indice de shock, Trauma.

How to cite this article: Ordonez CA, Orozco V, Puyana JC, Parra M, Ossa P, Escobar SS, Cevallos C, Garcia A, Badiel M, García MAM. Indice de Shock: ¿Puede Predecir La Necesidad de Cirugia de Conrol de Daños En Trauma Penetrante? Panam J Trauma Crit Care Emerg Surg 2017;6(2):72-76.

Source of support: Nil

Conflict of interest: None

\section{ABSTRACT}

Introduction: The objective of our study was to identify a possible shock index (SI) cutoff number that could reliable predicts the need for damage control surgery (DCS) in penetrating trauma patients.

Materials and methods: A retrospective 10-year study (20052015) which included all adult patients that suffered penetrating torso trauma at a level I trauma center. The SI was calculated upon admission and patients were divided into two groups: those that required DCS and those that did not (No-DCS). A $95 \%$ confidence interval for the area under a receiver operating characteristic curve (AUROC) was used to determine the accuracy of $\mathrm{SI}$ in detecting patients that would require DCS.

Results: A total of 747 patients were included, $92.3 \%$ were male and $80 \%$ suffered gunshot wounds. The DCS group had a total of $331(44 \%)$ patients and the No-DCS group had $416(56 \%)$. Mean SI values were higher in the DCS group (1.01 [0.76-1.5]) compare to the No-DCS (0.79[0.64-1.00]) ( $\mathrm{p}^{1 / 40.0001)}$. The AUROC was 0.69 [95\% Cl 0.69-0.77].

Conclusions: We have shown that SI can be indicative to some degree of the need for DCS in patients suffering penetrating torso trauma but a specific cutoff point that can precisely predict this need is statically unobtainable.

Keywords: Damage Control Surgery, Hemorrhage, Panamerican Tr Society, Penetrating trauma, Prediction, Shock index, Trauma.

\section{INTRODUCCIÓN}

El trauma es una de las principales causas de mortalidad a nivel mundial. ${ }^{1,2}$ La mayoría de estas muertes ocurren durante las primeras horas, principalmente a nivel prehospitalario. ${ }^{3}$ La evaluación inicial del paciente traumatizado se realiza con variables fisiológicas como la frecuencia cardiaca (FC) y la presión arterial media (PAM) entre otros, con el fin de identificar aquellos pacientes 
con inestabilidad hemodinámica, shock hemorrágico y/o necesidad de intervención inmediata. En el paciente con hemorragia oculta, cuyas constantes vitales se encuentran dentro de límites normales, el diagnosticar un shock hemorrágico ${ }^{4}$ inadvertido es un reto para el profesional en el triage.

Los pacientes con trauma severo penetrante exanguinados e inestabilidad hemodinámica son manejados con Cirugía de Control de Daños (CCD) por los beneficios evidenciados en los últimos años reflejados con el incremento en la sobrevida. ${ }^{5-8}$ Sin embargo la demora en la Sala de Urgencias (SU) para la identificación y tratamiento de un paciente con signos vitales (FC, PAS, PAM) normales pueden retrasar la decisión del cirujano en abordar al paciente con CCD en el escenario de un paciente con pobre oxigenación/perfusión secundario a hemorragia por trauma.

El índice de shock (IS), cociente entre la Presión arterial sistólica (PAS) y la FC, fue propuesto por primera vez en 1967 como una medida practica para estimar el grado de hipovolemia en el shock hemorrágico y séptico. ${ }^{9}$ Se ha propuesto este índice como un marcador del grado de shock, disminución en la oxigenación tisular y falla ventricular izquierda persistente durante el manejo del shock en (SU). ${ }^{10}$ Adicionalmente se ha usado en diferentes estudios para predecir mortalidad y requerimientos de transfusiones masiva en el paciente de trauma. ${ }^{11,12} \mathrm{El}$ objetivo de este estudio es analizar la habilidad del IS para predecir en pacientes con trauma penetrante de torso y encontrar un punto de corte como predictor de CCD.

\section{MATERIALES Y MÉTODOS}

Se realizó un estudio retrospectivo de 10 años (20052015) utilizando el registro de Trauma de la Fundación Valle del Lili el cual contiene variables demográficas, clínicas, relacionadas al trauma, índices de severidad, seguimiento y desenlaces. La Fundación Valle del Lili ubicada en Cali, Colombia, es una institución privada universitaria, Centro de Trauma Nivel I de referencia del Suroccidente del país para la atención en trauma a civiles y militares.

El presente estudio incluyo todos los pacientes con trauma penetrante abdominal y/o torácico que fueran llevados a Laparotomía y/o Toracotomía, con signos vitales tomados al ingreso a SU para calcular el SI, Base exceso, $\mathrm{pH}$, sangrado intraquirurgico y tiempo de UCI y hospitalización. Se excluyeron los pacientes con Trauma Craneoencefálico severo con un puntaje en la escala de Glasgow menor a 9 y los pacientes que llegaron sin signos vitales al Centro.

Los pacientes se dividieron en dos grupos: Aquellos que fueron llevados a CCD y los que no CCD (No-CCD). Se obtuvieron variables demográficas, fisiológicas (Base exceso, Ph, Temperatura), Soportes requeridos (transfusiones de PRBC durante cirugía, cristaloides en las primeras 24 horas), Índices de Severidad (ISS, NISS), días de estancia en Unidad de cuidado intensivo, estancia hospitalaria y mortalidad. La FC y PAS se obtuvo en el triage, y se calculo el IS usando la definición de IS=FC / PAS a todos los pacientes. El desenlace principal de este estudio es la CCD, por lo cual los pacientes se dividieron en dos grupos según la realización de este procedimiento.

Las variables numéricas con distribución normal se sintetizaron en medias y desviaciones estándar \pm y para su análisis se uso una prueba t de Student. La prueba U de Mann-Whitney se uso para comparar la información con distribución anormal, y se presento en medianas y rangos intercuartilicos [RIC]. Las variables categóricas se presentaron como proporciones y se compararon mediante una prueba exacta de Fisher o una prueba de Chi. ${ }^{2}$ Para determinar la exactitud y habilidad del IS en predecir la necesidad de CCD y encontrar un punto de corte se realizo un Intervalo de confianza del 95\% para el Area Under a Receiver Operating Characateristic Curve (AUROC). Para el análisis estadístico se utilizó STATA software (version 13). El estudio fue aprobado por el comité de ética del hospital

\section{RESULTADOS}

Un total de 747 pacientes se incluyeron, el 92.3\% fueron hombres y la mediana de edad fue 27 [22-36 IQR] años. El principal mecanismo de trauma fue herida por arma de fuego (HPAF) en el 80\% (597) de los pacientes, seguido por las heridas por Arma Cortopunzante (HPAC) 18\% (137) y explosiones en el 1\%. ${ }^{11}$ Al 44\% (331) de los pacientes se les realizo CCD y el 56\% (416) conformo el grupo de No-CCD, las principales características demográficas de ambos grupos se encuentran resumidas en la tabla 1.

El procedimiento más frecuente en ambos grupos fue la laparotomía, $89 \%$ (295) vs. 76\% (317) en CCD y No-CCD

Tabla 1: Características demográficas de los pacientes

\begin{tabular}{llll}
\hline & $C C D n=331$ & No-CCD $n=416$ & $p$-valor \\
\hline $\begin{array}{l}\text { Edad, mediana } \\
\text { [RIC] }\end{array}$ & $27(22-36)$ & $27(21.5-37)$ & 0.983 \\
$\begin{array}{l}\text { Sexo, } \mathrm{n}(\%) \\
\quad \text { Masculino }\end{array}$ & $306(92.45 \%)$ & $384(92.31 \%)$ & 0.912 \\
$\begin{array}{l}\text { Mecanismo de } \\
\text { Trauma, } \mathrm{n}(\%)\end{array}$ & & & \\
$\quad$ HPAF & $288(87 \%)$ & $309(74 \%)$ & $<0.0001$ \\
$\quad$ HPAC & $35(11 \%)$ & $102(25 \%)$ & $<0.0001$ \\
$\quad$ Explosión & $7(2 \%)$ & $4(1 \%)$ & $<0.442$ \\
\hline
\end{tabular}

CCD: Cirugía de Control de Daños, RIC: Rango Intercuartilico, HPAF: Herida por arma de fuego, HPAC: Herida por arma cortopunzante 


\begin{tabular}{lllr}
\hline \multicolumn{4}{c}{ Tabla 2: Lesiones Asociadas, n (\%) } \\
\hline & $C C D n=331$ & No-CCD $n=416$ & $p$-valor \\
\hline Víscera Hueca & $226(68 \%)$ & $194(47 \%)$ & $<0.0001$ \\
Órgano Solido & $175(53 \%)$ & $123(30 \%)$ & $<0.0001$ \\
Grandes Vasos & $93(28 \%)$ & $49(12 \%)$ & $<0.0001$ \\
\hline
\end{tabular}

CCD: Cirugía de Control de Daños

Tabla 3: Índices de Severidad

\begin{tabular}{lllr}
\hline Índices, mediana [RIC] & $C C D$ & No-CCD & $p$-valor \\
\hline ISS & $25(16-29)$ & $16(9-22)$ & $<0.0001$ \\
NISS & $34(25-50)$ & $25(10-34)$ & $<0.0001$ \\
\hline
\end{tabular}

respectivamente. Seguido por la toracotomía 33\% (110) y $29 \%$ (120) y los procedimientos combinados (Laparotomía y Toracotomía), que fueron más frecuentes en el grupo de CCD (74 (22\%) vs. $21(5 \%)$ p $<0.001)$. En los pacientes con control de daños, el 73\% quedaron empaquetados, ya sea del abdomen, tórax o ambas cavidades.

Las variables fisiológicas asociadas a un estado de acidosis y menor perfusión tisular fueron el $\mathrm{pH}$ (7.27 [7.18-7.34] vs 7.31 [7.27-7.36] $\mathrm{p}<0.0001)$, la Base exceso $(-9.3[-12$ to -6.2$]$ vs. $-6.9[-9.3$ to -5.1$] \mathrm{p}<0.0001)$ y el lactato (3.7 [2.6-5.8] vs 2.4 [1.7-3.9] $\mathrm{p}<0.0001)$ en CCD y No-CCD respectivamente.

Dentro de los hallazgos intraoperatorios la lesión de víscera hueca la lesión mas encontrada en ambos grupos (68\% y 47\%). Los demás patrones de lesión se encuentran resumidos en la tabla 2.

La mediana del sangrado intraquirurgico fue en general de 1500 [800-3000] cc, sin embargo en el grupo de CCD la mediana fue de 2500 [1500-4000] cc y en el grupo No-CCD fue de 1000 [500-1850] cc. Las PRBC en cirugía fueron de 4 [2-8] unidades y de 2 [2-5] unidades en cada grupo. Los cristaloides requeridos en las primeras 24 horas en el grupo CCD fueron de 6125 [3872-9025] cc, mientras que en los pacientes de No-CCD fue de 3600 [2500-5000] cc. Los índices de severidad se encuentran resumidos en la tabla 3.

Los principales desenlaces en cada grupo están resumidos en la tabla 4. La mortalidad en el grupo de CCD fue del $20 \%$.

El índice de shock fue mayor en el grupo de CCD (1.01 [0.76-1.5]) comparado con No-CCD (0.79 [0.64-1.00]) $(\mathrm{p}=0.0001)$. El AUROC para el IS fue de 0.69 [95\% CI 0.690.77] (Grafica 1) sin evidenciar un punto de corte con una sensibilidad o especificidad suficiente para predecir la variable desenlace principal, cuando se usaba un punto de corte del IS de 0.9 , con exactitud clasificaba al 64.7\% de los pacientes que requerían $C C D$, con una sensibilidad del $63.3 \%$, especificidad del $65.8 \%$ y una tasa de falsos negativos del $36.7 \%$.
Tabla 4: Resultados de pacientes con trauma penetrante

\begin{tabular}{llll}
\hline & $\begin{array}{l}C D \\
n=331\end{array}$ & $\begin{array}{l}\text { No-CCD } \\
n=416\end{array}$ & $p$-valor \\
\hline $\begin{array}{l}\text { Días en UCI, } \\
\text { mediana (RIC) }\end{array}$ & $7(4-12.5)$ & $3(2-5.5)$ & 0.0001 \\
Días totales de & $11(5-20)$ & $5(3-9)$ & 0.0001
\end{tabular}

hospitalización,

mediana (RIC)

Mortalidad, n (\%) $\quad 69(20.85 \%) \quad 16(3.85 \%) \quad 0.0001$

RIC: Rango intercuartilico, UCl: Unidad de Cuidado Intensivo

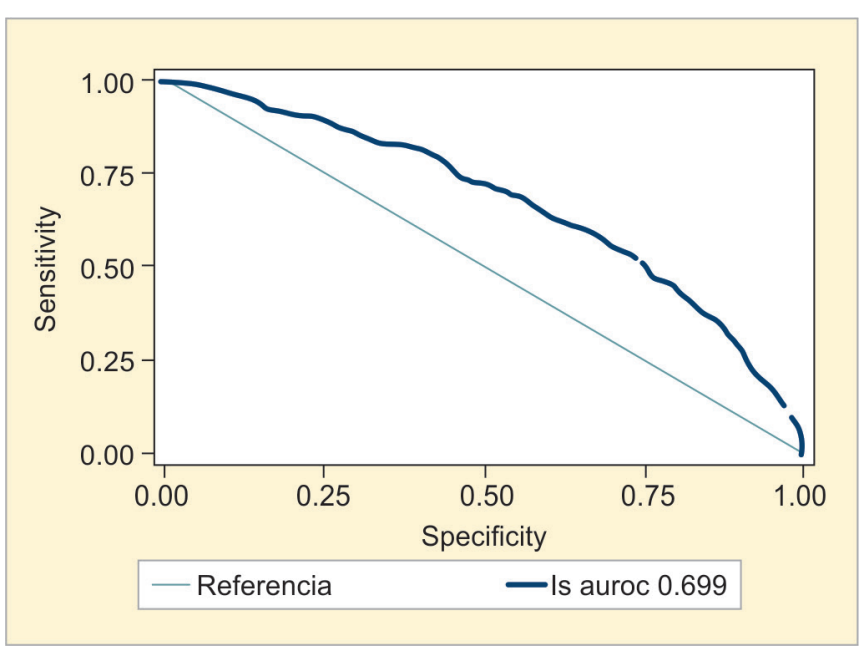

Grafica 1: Curva ROC del Índice de Shock y la realización de Cirugía de Control de daños

\section{DISCUSIÓN}

Durante este estudio se intento encontrar un punto de corte del IS para predecir la necesidad de CCD, encontrándose una diferencia estadísticamente significativa entre el grupo de CCD y No-CCD, sin embargo a pesar de existir una relación entre el IS y la necesidad de CCD, no fue posible encontrar un punto de corte con adecuada discriminación. El IS se ha usado en estudios previos para predecir mortalidad y requerimiento de transfusiones en el paciente traumatizado, dos cosas comunes en el Control de Daños. En el 2009 se publico un estudio en el cual se asocio los pacientes con un IS mayor a 0.9 (RR 1.6 IC $95 \%$ 1.13-2.33) con las transfusiones masivas, y en los pacientes con un IS mayor de 1.1 a 1.3 este riesgo incrementado 5 veces y hasta 8 veces en los pacientes con un IS mayor a 1.3. ${ }^{13}$ Un metaanálisis publicado en el 2013 busco la relación entre los signos vitales y el sangrado activo, incluyo 30 estudios, la FC, PAS e IS fueron los parámetros más estudiados, y encontraron que los estudios que usaban una curva ROC para analizar los datos, se encontró un AUROC de 0.77 a 0.84 en aquellos que incluyeron al IS. ${ }^{14}$ Ellos resaltan que el IS puede ser un indicador preciso de cambios compensatorios en el sistema cardiovascular secundario a la hemorragia. ${ }^{14} \mathrm{El}$ índice de shock ya se ha relacionado con la detección temprana 
de la hemorragia, demostrando un mejor rendimiento que la Frecuencia cardiaco o la Presión arterial sistólica por si solas. ${ }^{15}$ En ambos grupos se evidencio un índice de shock anormal $(>0.7)$ y con evidencia de inestabilidad hemodinámica $(>1.0) \cdot{ }^{16}$ Sin embargo no existe ninguna publicación en la cual se haya intentado usar este índice para predecir CCD, el resultado del estudio a pesar de la asociación de estudios previos del IS a los pacientes más críticos y severos victimas del trauma, no es suficientemente fuerte para encontrar un punto de corte, por lo cual no es candidato para ser incluido en protocolos de Trauma, a pesar de evidenciarse un IS mayor en los pacientes con CCD.

La hemorragia por trauma es una de las principales causas de muerte, en un $30 \%$ a $40 \%$ de todas las muertes ocasionadas por trauma. ${ }^{17} \mathrm{La}$ importancia del control de la hemorragia, lleva a darle un enfoque multidisciplinario que implica el uso de estrategias que incluyen Cirugía de Control de daños como una herramienta junto con la resucitación hemostática, la hipotensión permisiva, el uso restringido de cristaloides y la corrección de la hipotermia. ${ }^{18,19}$ Evidenciar la hemorragia tempranamente y definir si el paciente es candidato para CCD facilitaría toma de decisiones por parte del cirujano y de equipo de trauma La letalidad de la hemorragia la convierte en uno de los principios de CCD "Control temprano de la Hemorragia" 20 junto con el manejo de la contaminación y el empaquetamiento, entre otras, es por esto que la estimación del sangrado se considera una buena medida para tomar decisiones, sin embargo limitada en el caso de que el sangrado no sea externo. En el caso de la atención del paciente en el campo prehospitalario, se han desarrollado varios criterios de triage para determinar la severidad de las lesiones, el modo de transporte y el destino más apropiado para un paciente de trauma. ${ }^{21-23}$ En este campo el índice de shock ha sido propuesto como un criterio en el Protocolo Nacional de Triage en Trauma en los Estados Unidos para direccionar al el paciente a un Centro de Trauma, mostrando que un valor $>1.0$ aumenta la sensibilidad con una mínima reducción de la especificidad comparado con una tensión arterial sistólica $<90 \mathrm{mmHg}$ para identificar a los pacientes inestables que requieren atención de mayor complejidad. ${ }^{24}$

A pesar de tener un IS superior en el grupo de CCD, creemos que la poca habilidad discriminatoria de este índice está influenciada por varios factores: el primero de ellos es que la toma de los signos vitales para el cálculo del IS se realizo en un intervalo de tiempo entre el ingreso del paciente a urgencias hasta el ingreso a salas de cirugía, lo cual puede haber variado debido al agravamiento del shock hemorrágico, por esto algunos pacientes que posterior a la toma de signos se inestabilizados fueron llevados a CCD y su IS vario en ese lapso de tiempo, pensando en aquellos estudios en los cuales se ha comparado el IS prehospitalario con el hospitalario para predecir desenlaces y ha mostrado mayor capacidad el prehospitalario. ${ }^{12}$ Otra hipótesis son aquellos pacientes que pueden funcionar como factor de confusión, como es el caso de los pacientes con trauma cardiaco o trauma pulmonar, exanguinados, con un IS severo y al realizarse la cardiorrafia o la neumorrafia, mejora su hemodinamia sin requerir procedimiento abreviado y un segundo procedimiento definitivo, estos pacientes críticos pero que no requirieron $C C D$ pueden alterar este resultado.

Las limitaciones de este estudio incluyen su diseño retrospectivo, representa los datos de una sola institución. Adicionalmente, la potencial introducción de sesgo de selección ante la ausencia de un protocolo establecido para la realización de CCD o no, lo cual recayó sobre el criterio del cirujano. Sin embargo, este diseño simula las condiciones reales del entorno clínico donde son los cirujanos quienes finalmente basados en su criterio clínico toman una decisión. La estrategia de orientar las intervenciones a optimizar el estado fisiológico del paciente durante la "hora dorada" posterior al trauma, incluyen la reducción del tiempo en el transporte prehospitalario, los protocolos de transfusiones masivas y la cirugía, en el caso del paciente con un compromiso mayor, Control de Daños, ${ }^{25,26}$ son la piedra angular para optimizar la sobrevida de los pacientes con hemorragia exanguinante. En este caso para tratar de ganar tiempo, se intento desarrollar una medida que predijera el requerimiento de control de daños, sin embargo su habilidad es limitada para este objetivo, lo cual implica que la decisión de la conducta con el paciente crítico en trauma, no es estandarizada, y depende de otros factores adicionales a la inestabilidad hemodinámica y a lo reflejado por la alteración del sistema cardiovascular. La poca habilidad del IS hace difícil establecer un punto de corte que pueda usarse como parámetro para tomar alguna decisión sobre la CCD, al parecer nada puede reemplazar el juicio y el ejercicio analítico sobre cada paciente que realiza el cirujano.

\section{REFERENCES}

1. Peden M, McGee K, Sharma G. The injury chart book: a graphical overview of the global burden of injuries [Internet]. Geneva, World Heal Organ; 2002. pp. 6-76. Available from: http://www.who.int/iris/handle/10665/42566

2. World Health Organization. Global Status Report on Violence Prevention 2014 [Internet]. World Heal Organ; 2014. pp. 1-274. Available from: http://www.who.int/violence_injury_prevention/violence/status_report/2014/en/

3. Acosta JA, Yang JC, Winchell RJ, Simons RK, Fortlage DA, Hollingsworth-Fridlund P, Hoyt DB. Lethal injuries and time to death in a level I trauma center. J Am Coll Surg 1998 May;186(5):528-533.

4. Gutierrez G, Reines HD, Wulf-Gutierrez ME. Clinical review: hemorrhagic shock. Crit Care 2004;8(5):373-381. 
5. Hirshberg A, Wall MJ Jr, Mattox KL. Planned reoperation for trauma: a two year experience with 124 consecutive patients. J Trauma 1994 Sep;37(3):365-369.

6. Saifi J, Fortune JB, Graca L, Shah DM. Benefits of intra-abdominal pack placement for the management of nonmechanical hemorrhage. Arch Surg 1990 Jan;125(1):119-122.

7. Rotondo MF, Schwab CW, McGonigal MD, Phillips GR 3rd, Fruchterman TM, Kauder DR, Latenser BA, Angood PA. "Damage control": an approach for improved survival in exsanguinating penetrating abdominal injury. J Trauma 1993 Sep;35(3):373-375.

8. Shapiro MB, Jenkins DH, Schwab W, Rotondo MF. Damage Control: Collective Review. J Trauma 2000;49(5): 969-978.

9. Allgower M, Burri C. ["Shock index"]. Dtsch Med Wochenschr 1967 Oct;92(43):1947-1950. (German)

10. Rady MY, Rivers EP, Martin GB, Smithline H, Appelton T, Nowak RM. Continuous central venous oximetry and shock index in the emergency department: use in the evaluation of clinical shock. Am J Emerg Med 1992 Nov;10(6): 538-541.

11. Montoya KF, Charry JD, Calle-Toro JS, Núñez LR, Poveda G. Shock index as a mortality predictor in patients with acute polytrauma. J Acute Dis 2015;4(3):202-204.

12. Olaussen A, Peterson EL, Mitra B, O'Reilly G, Jennings PA, Fitzgerald M. Massive transfusion prediction with inclusion of the pre-hospital Shock Index. Injury. 2015 May;46(5): 822-826.

13. Vandromme MJ, Griffin RL, Kerby JD, McGwin GJ, Rue LW 3rd, Weinberg JA. Identifying risk for massive transfusion in the relatively normotensive patient: utility of the prehospital shock index. J Trauma 2011 Feb;70(2):384-390.

14. Pacagnella RC, Souza JP, Durocher J, Perel P, Blum J, Winikoff B, Gülmezoglu AM. A systematic review of the relationship between blood loss and clinical signs. PLoS One 2013;8(3): e57594.

15. Birkhahn RH, Gaeta TJ, Terry D, Bove JJ, Tloczkowski J. Shock index in diagnosing early acute hypovolemia. Am J Emerg Med 2005 May;23(3):323-326.
16. Rady MY, Nightingale P, Little RA, Edwards JD. Shock index: a re-evaluation in acute circulatory failure. Resuscitation 1992;23(3):227-234.

17. Kauvar DS, Lefering R, Wade CE. Impact of hemorrhage on trauma outcome: an overview of epidemiology, clinical presentations, and therapeutic considerations. J Trauma 2006;60(Suppl 6):S3-S11.

18. Duchesne JC, McSwain NE Jr, Cotton BA, Hunt JP, Dellavolpe J, Lafaro K, Marr AB, Gonzalez EA, Phelan HA, Bilski T. Damage control resuscitation: the new face of damage control J Trauma 2010 Oct;69(4):976-990.

19. Morrison JJ, Rasmussen TE. Noncompressible Torso Hemorrhage. A Review with Contemporary Definitions and Management Strategies. Surg Clin North Am 2012;92(4):843-858.

20. Finlay IG, Edwards TJ, Lambert AW. Damage control laparotomy. Br J Surg 2004 Jan;91(1):83-85.

21. Crystal R, Bleetman A, Steyn R. Ambulance crew assessment of trauma severity and alerting practice for trauma patients brought to a general hospital. Resuscitation 2004 Mar;60(3):279-282.

22. Koehler JJ, Baer LJ, Malafa SA, Meindertsma MS, Navitskas NR, Huizenga JE. Prehospital Index: a scoring system for field triage of trauma victims. Ann Emerg Med 1986 Feb;15(2):178-182.

23. Ocak G, Sturms LM, Hoogeveen JM, Le Cessie S, Jukema GN. Prehospital identification of major trauma patients. Langenbecks Arch Surg 2009 Mar;394(2):285-292.

24. Haider AA, Azim A, Rhee P, Kulvatunyou N, Ibraheem K, Tang A, O'Keeffe T, Iftikhar H, Vercruysse G, Joseph B. Substituting systolic blood pressure with shock index in the National Trauma Triage Protocol. J Trauma Acute Care Surg 2016 Dec;81(6):1136-1141.

25. Kotwal RS, Howard JT, Orman JA, Tarpey BW, Bailey JA, Champion HR, Mabry RL, Holcomb JB, Gross KR. The Effect of a Golden Hour Policy on the Morbidity and Mortality of Combat Casualties. JAMA Surg 2016 Jan;151(1):15-24.

26. Waters JH. Role of the massive transfusion protocol in the management of haemorrhagic shock. Br J Anaesth 2014 Dec;113(Suppl 2):ii:3-8. 